\title{
The Radio-bright Accreting Millisecond X-Ray Pulsar IGR J17591-2342
}

\author{
T. D. Russell ${ }^{1}$ (i), N. Degenaar ${ }^{1}$, R. Wijnands ${ }^{1}$, J. van den Eijnden ${ }^{1}$, \\ N. V. Gusinskaia ${ }^{1,2}$, J. W. T. Hessels ${ }^{1,2}$ (iD), and J. C. A. Miller-Jones ${ }^{3}$ (iD \\ ${ }^{1}$ Anton Pannekoek Institute for Astronomy, University of Amsterdam, Science Park 904, 1098 XH, Amsterdam, The Netherlands; t.d.russell@uva.nl \\ ${ }^{2}$ ASTRON, Netherlands Institute for Radio Astronomy, Oude Hoogeveensedijk 4, 7991 PD, Dwingeloo, The Netherlands \\ ${ }^{3}$ International Centre for Radio Astronomy Research-Curtin University, GPO Box U1987, Perth, WA 6845, Australia \\ Received 2018 October 31; revised 2018 November 28; accepted 2018 November 29; published 2018 December 11
}

\begin{abstract}
IGR J17591-2342 is a $527 \mathrm{~Hz}$ accreting millisecond X-ray pulsar that was discovered in outburst in 2018 August. In this Letter, we present quasi-simultaneous radio and X-ray monitoring of this source during the early part of the outburst. IGR J17591-2342 is highly absorbed in X-rays, with an equivalent hydrogen absorption along the line of sight, $N_{\mathrm{H}}$, of $\approx 4.4 \times 10^{22} \mathrm{~cm}^{-2}$, where the Galactic column density is expected to be $\approx 1-2 \times 10^{22} \mathrm{~cm}^{-2}$. The high absorption suggests that the source is either relatively distant $(>6 \mathrm{kpc})$, or that the X-ray emission is strongly absorbed by material local to the system. Radio emission detected by the Australia Telescope Compact Array shows that, for a given X-ray luminosity and for distances greater than $3 \mathrm{kpc}$, this source was exceptionally radio-loud when compared to other accreting neutron stars in outburst $\left(L_{\mathrm{X}}>10^{33} \mathrm{erg} \mathrm{s}^{-1}\right)$. For most reasonable distances, IGR J17591-2342 appeared as radio luminous as actively accreting, stellar-mass black hole X-ray binaries.
\end{abstract}

Key words: accretion, accretion disks - ISM: jets and outflows - pulsars: general - radio continuum: stars - stars: neutron - X-rays: binaries

\section{Introduction}

Accreting millisecond X-ray pulsars (AMXPs) are binary systems in which a neutron star (NS) primary accretes material -often only sporadically-from a low-mass companion star. However, in contrast to the majority of known neutron star X-ray binaries (NSXRBs), these objects exhibit X-ray pulsations as material is channeled from the disk onto the magnetic poles of the rotating NS (e.g., Wijnands \& van der Klis 1998). These objects are thought to be the link between accreting lowmass X-ray binaries (XRBs) and millisecond pulsars (MSPs; see Wijnands \& van der Klis 1998; Patruno \& Watts 2012, for review) where the transfer of angular momentum during the accreting XRB phase can spin up the NS to millisecond periods (e.g., Alpar et al. 1982; Radhakrishnan \& Srinivasan 1982). This link has been exemplified, e.g., by the systems PSR J1023 +0038 and M28I (IGR J18245-2452), which have been observed to transition between radio MSP and AMXP states on relatively short timescales (Archibald et al. 2009; Papitto et al. 2013; Stappers et al. 2014). Such transitional MSPs (tMSPs) are also thought to switch between accretion-powered and rotation-powered pulsar states.

AMXPs are typically transient, going through cycles of outburst and quiescence (e.g., in 't Zand et al. 1998). During outburst, radio emission with a flat or inverted spectrum $(\alpha \approx 0$, where the flux density, $S_{\nu}$, scales with the frequency, $\nu$, such that $S_{\nu} \propto \nu^{\alpha}$ ) is observed (e.g., Tudor et al. 2017), which is indicative of a partially self-absorbed, compact jet (e.g., Blandford \& Königl 1979).

In their hard X-ray states at the beginning and end of a typical outburst, black hole XRBs (BHXRBs) exhibit a tight, non-linear relationship between their radio $\left(L_{\mathrm{R}}\right)$ and X-ray $\left(L_{\mathrm{X}}\right)$ luminosities that extend over several decades (e.g., Gallo et al. 2012; Corbel et al. 2013). The observed empirical relationship is generally described by two distinct power-law tracks in the radio/X-ray luminosity plane; an upper "radio-loud" track with a best-fitting slope of $L_{\mathrm{R}} \propto L_{\mathrm{X}}{ }^{0.6}$ and a lower, steeper, "radio-quiet" track with a best-fitting slope of $L_{\mathrm{R}} \propto L_{\mathrm{X}}^{\sim}$ (Gallo et al. 2012). However, recently the statistical significance of the two separate tracks has been questioned (Gallo et al. 2014, 2018). Also, individual systems have shown significant deviation over luminosity ranges spanning $<2$ orders of magnitude (e.g., Corbel et al. 2013).

For accreting NSs the picture is even less clear. Not only is the connection complicated by the presence of a stellar surface/ magnetosphere (e.g., Maccarone 2008), but NSs also appear fainter in the radio than their BH counterparts (e.g., Fender \& Kuulkers 2001; Migliari \& Fender 2006), meaning that their $L_{\mathrm{R}} / L_{\mathrm{X}}$ behavior has generally been measured across a smaller dynamic range (and with lower detection significance) than their BH counterparts. Initially, a steep $\sim 1.4$ correlation was suggested (Migliari \& Fender 2006). However, individual systems appear to show varying correlation indices and normalizations, and not all follow similar, or even welldefined, tracks (e.g., Tudor et al. 2017). To try to account for the restricted luminosity ranges typically measured for NS systems, regression analysis of a large sample of NS systems showed that as a whole they appear to be a factor of $\sim 22$ fainter than the population of hard-state BHs, but with a similar correlation slope (Gallo et al. 2018).

The differences in both normalization and slope for individual systems could be driven by a number of factors, such as jet power, primary mass, spin, magnetic field, and jetlaunching mechanism. Recent results showing faint radio jets being launched from a slowly spinning high-magnetic-field $\left(>10^{12} \mathrm{G}\right)$ accreting NS indicate that the magnetic field or spin of the NS could be playing an important role in the radio brightness (van den Eijnden et al. 2018a).

\subsection{IGR J17591-2342}

IGR J17591-2342 was discovered in outburst by the INTErnational Gamma-Ray Astrophysics Laboratory (INTEGRAL) on 2018 August 10-11 (MJD 58340-58341), during monitoring of the Galactic center (Ducci et al. 2018). Archival all-sky Neil 
Table 1

ATCA Radio Observations of IGR J17591-2342

\begin{tabular}{ccccc}
\hline \hline Date & MJD & $\begin{array}{c}\text { Frequency } \\
(\mathrm{GHz})\end{array}$ & $\begin{array}{c}\text { Flux Density } \\
(\mathrm{mJy})\end{array}$ & $\alpha$ \\
\hline 2018 & $58344.56 \pm 0.12$ & 5.5 & $1.09 \pm 0.02$ & $0.1 \pm 0.3$ \\
Aug 14 & & 9.0 & $1.12 \pm 0.02$ & \\
& & 5.5 & $1.00 \pm 0.05$ & $-0.30 \pm 0.25$ \\
2018 & $58349.26 \pm 0.07$ & & & \\
Aug 19 & & 9.0 & $0.85 \pm 0.05$ & \\
& & 5.5 & $1.18 \pm 0.04$ & $0.25 \pm 0.1$ \\
2018 & $58355.28 \pm 0.12$ & & & \\
Aug 25 & & 9.0 & $1.21 \pm 0.04$ & \\
& & 17.0 & $1.54 \pm 0.10$ & \\
& $58355.28 \pm 0.10$ & 19.0 & $1.60 \pm 0.09$ & \\
& & & & \\
\hline
\end{tabular}

Note. MJDs note the mid-point of the observation, with the quoted uncertainties reflecting the observation duration.

Gehrels Swift Observatory (Swift) Burst Alert Telescope (BAT) observations indicate that the outburst rose above detection threshold around 2018 July 22 (MJD 58321), 20 days earlier (Krimm et al. 2018). We observed the source at radio wavelengths using the Australia Telescope Compact Array (ATCA), detecting bright radio emission, leading to our initial suggestion that the source may be a BH candidate (Russell et al. 2018). However, coherent X-ray pulsations at $527.4 \mathrm{~Hz}(1.9 \mathrm{~ms})$ detected using the Nuclear Spectroscopic Telescope Array (NuSTAR) and the Neutron star Interior Composition Explorer (NICER) identified this source as an AMXP (Sanna et al. 2018), with an orbital period of $\sim 8.8 \mathrm{hr}$ and a companion mass of $\gtrsim 0.42 M_{\odot}$. Assuming a $1.4 M_{\odot}$ NS mass and typical AMXP parameters, the spin frequency derivative implies a magnetic field of $1.4 \times$ $10^{8}<B<8 \times 10^{9} \mathrm{G}$ (Sanna et al. 2018). After discovery, the source stayed active for $\sim 80$ days, exhibiting some long-term variability, including multiple re-brightenings.

In this Letter, we discuss quasi-simultaneous radio and X-ray observations taken in the early stages of the outburst (spanning MJDs 58344-58356). The high X-ray absorption suggests that the source is relatively distant and therefore much more radio luminous than that from other known NS systems. We investigate the implications of this radio brightness in the context of other known XRBs.

\section{Observations}

\subsection{ATCA Radio Observations}

We conducted DDT radio observations (project ID: CX413) with the Australia Telescope Compact Array (ATCA). IGR J17591-2342 was observed three times early in its outburst, on 2018 August 14, 19, and 25 (MJDs 58344, 58349 , and 58355, respectively; see Table 1). The observations were taken at central frequencies of 5.5 and $9 \mathrm{GHz}$. The August 25 observations had additional frequencies of 17 and $19 \mathrm{GHz}$. Each frequency pair was taken simultaneously. All bands were recorded with a bandwidth of $2 \mathrm{GHz}$. PKS 1934-638 was used for flux calibration, while phase calibration was carried out using 1817-254 on August 14 and with 1752-225 on August 19 and 25 . The data were flagged and calibrated following standard procedures within the Common Astronomy Software Applications (CASA v4.7; McMullin et al. 2007).

For the August 14 observation the telescope was in a relatively extended (1.5D) configuration. Imaging was carried out with Briggs weighting (robust $=0$ ), minimizing effects from diffuse emission in the field due to its location toward the Galactic center, providing angular resolutions of 6 "! $8 \times 1$ "! 4 at $5.5 \mathrm{GHz}$ and $3 . \prime 9 \times 0 . \prime 9$ at $9 \mathrm{GHz}$ (with a position angle 15 degrees north of east). The flux density was determined by fitting for a point source in the image plane using IMFIT. We measure a $(9 \mathrm{GHz})$ right ascension (R.A.) and declination (decl.) of

$$
\begin{aligned}
& \text { R.A.(J2000) }=17^{\mathrm{h}} 59^{\mathrm{m}} 02^{\mathrm{s}} .86+/-0.04, \\
& \text { decl.(J2000) }=-23^{\circ} 43^{\mathrm{m}} 08^{\mathrm{s}}: 3+/-0.1,
\end{aligned}
$$

where the uncertainties are from the fitted position of the point source, which are larger than the theoretical statistical error from beam centroiding.

For our August 19 and 25 observations, ATCA was in a compact (H75) configuration, where five of the six antennas are located within 75 meters of each other. Due to significant diffuse emission, imaging was not trivial. However, ATCA's sixth antenna is located $6 \mathrm{~km}$ from the array core. Using only the longest baselines out to antenna six to eliminate issues from diffuse emission, we determined the flux by fitting for all sources in the field in the uv-plane using UVMULTIFIT (Martí-Vidal et al. 2014).

See Table 1 for source flux densities.

\subsection{Swift X-Ray Observations}

The Swift X-ray Telescope (XRT) observed IGR J17591 -2342 every few days during the outburst (target IDs: 10803 and 10804). Observations closest in time to our ATCA monitoring (within 1 day) were extracted with the Swift-XRT online pipeline (Evans et al. 2009). We used the Swift-XRT online pipeline to create the light curve. The observations were conducted in photon counting (PC) mode.

To determine the X-ray flux of IGR J17591-2342 for epochs that were closest to the radio observation, we analyzed the $0.5-10 \mathrm{keV}$ spectra with XSPEC version 12.9 (Arnaud 1996). The data were best fit with an absorbed (Wilms et al. 2000) power-law model; Tbabs $\times$ powerlaw (C-stat/ degrees of freedom $=269 / 240$ ). The addition of a thermal component did not improve the fits, or change the results. $\mathrm{X}$-ray fluxes were determined using the XSPEC convolution model cflux. Results are shown in Table 2. The equivalent hydrogen column density, $N_{\mathrm{H}}$, was tied between epochs (determined by jointly fitting all 17 Swift observations between MJDs 58342-58403). Leaving $N_{\mathrm{H}}$ free gave similar results for all but the August 18 observation, where the low count rate meant poor model constraints. Fixing $N_{\mathrm{H}}$ to significantly different values produced poor fits and unphysical results.

\section{Results and Discussion}

\subsection{Radio and X-Ray Light Curves}

Following its initial X-ray detection, IGR J17591-2342 first brightened for a few days before reaching an initial X-ray peak 
Table 2

Best-fitting Spectral Parameters for the 0.5-10 keV Swift-XRT Observations that were Closest in Time to the ATCA Radio Observations

\begin{tabular}{lccccc}
\hline \hline Date & MJD & Obs ID & $\Gamma$ & $\begin{array}{c}\text { Absorbed } \\
1-10 \mathrm{keV} \mathrm{Flux} \\
\left(\times 10^{-10}\right) \mathrm{erg} \mathrm{s}^{-1} \mathrm{~cm}^{-2}\end{array}$ \\
\hline 2018 Aug 14 & $58344.688 \pm 0.002$ & 00010804002 & $1.55 \pm 0.15$ & $1.98_{-0.15}^{+0.16}$ & $\begin{array}{c}\text { Unabsorbed } \\
1-10 \mathrm{keV} \mathrm{Flux} \\
\left(\times 10^{-10}\right) \mathrm{erg} \mathrm{s}^{-1} \mathrm{~cm}^{-2}\end{array}$ \\
2018 Aug 18 & $58348.223 \pm 0.003$ & 00010804004 & $2.1 \pm 0.3$ & $0.79_{-0.09}^{+0.11}$ & $2.96_{-0.16}^{+0.17}$ \\
2018 Aug 25 & $58355.559 \pm 0.033$ & 00010804006 & $2.0 \pm 0.15$ & $1.84 \pm 0.09$ & $3.30 \pm 0.13$ \\
\hline
\end{tabular}

Note. The data were fit with Tbabs $\times$ powerlaw within XSPEC. MJDs correspond to the mid-point of the X-ray observation (errors represent the observation duration). The column density along the line of sight, $N_{\mathrm{H}}$, was tied between epochs to give $N_{\mathrm{H}}=(4.4 \pm 0.3) \times 10^{22} \mathrm{~cm}^{-2}$. $\Gamma$ is the photon index of the power-law component. Fluxes were calculated using cflux. Errors are 1- $\sigma$.

around August 14 (MJD 58344), around the time of the first Swift-XRT observation. IGR J17591-2342 then faded steadily over the next $\sim 5$ days (Figure 1). The source then rebrightened, returning to a similar X-ray count rate as its initial $\mathrm{X}$-ray peak, following which it remained detectable for the next $\sim 80$ days.

A few days after the initial X-ray peak, we conducted our first radio observation, detecting the source. Over the next 2 weeks, we observed IGR J17591-2342 a further two times. During these observations, the source showed a similar evolution as the X-rays, where the radio flux faded briefly before re-brightening (Figure 1).

During our first ATCA observation, the radio spectrum was consistent with flat (Figure 1 and Table 1). The spectrum steepened slightly at the time of our second radio observation, although signal to noise and frequency coverage meant poor constraints on the spectrum. During our final radio observation, we observed a significantly inverted radio spectrum. These observations imply partially self-absorbed synchrotron emission from a compact jet (e.g., Fender 2001).

\subsection{Location on the Radio/X-Ray Plane}

To investigate the $L_{\mathrm{R}}$ and $L_{\mathrm{X}}$ of IGR J17591-2342, we place the $5 \mathrm{GHz}$ radio and $1-10 \mathrm{keV} \mathrm{X}$-ray luminosities on the radio/ $\mathrm{X}$-ray plane using several assumed distances (Figure 2). Unfortunately, our contemporaneous observations sample only a very limited range of $L_{\mathrm{R}}$ and $L_{\mathrm{X}}$ (factors of only $\sim 1.1$ and $\sim 2$, respectively). Therefore, while we are unable to determine the specific scaling between the $L_{\mathrm{R}}$ and $L_{\mathrm{X}}$ with this monitoring, the radio brightness is interesting.

In terms of its radio and X-ray luminosities, for distances greater than $\sim 3 \mathrm{kpc}$ IGR J17591-2342 would be brighter in the radio than the majority of other accreting NS systems (Figure 2). At a such distances, its $L_{\mathrm{R}} / L_{\mathrm{X}}$ ratio places IGR J17591-2342 in the region of parameter space occupied by BHXRBs. These results imply that IGR J17591-2342 was either considerably radio-loud during the early stages of its 2018 outburst, or it is closer than $3 \mathrm{kpc}$.

Comparing the observed radio emission from IGR J17591 -2342 to the sample of AMXPs with reported radio detections (Figure 2, magenta squares), we see that IGR J17591-2342 appears to be more radio bright than all other NS systems for distances greater than $\sim 5 \mathrm{kpc}$; this reduces to $\sim 3 \mathrm{kpc}$ without the inclusion of the AMXP IGR J17379-3747. The recent radio detection of IGR J17379-3747 (van den Eijnden et al. 2018 b) indicates that this is another potentially radio-bright AMXP (Figure 2, magenta star), where an $8 \mathrm{kpc}$ distance upper limit was estimated from type I X-ray bursts, assuming the

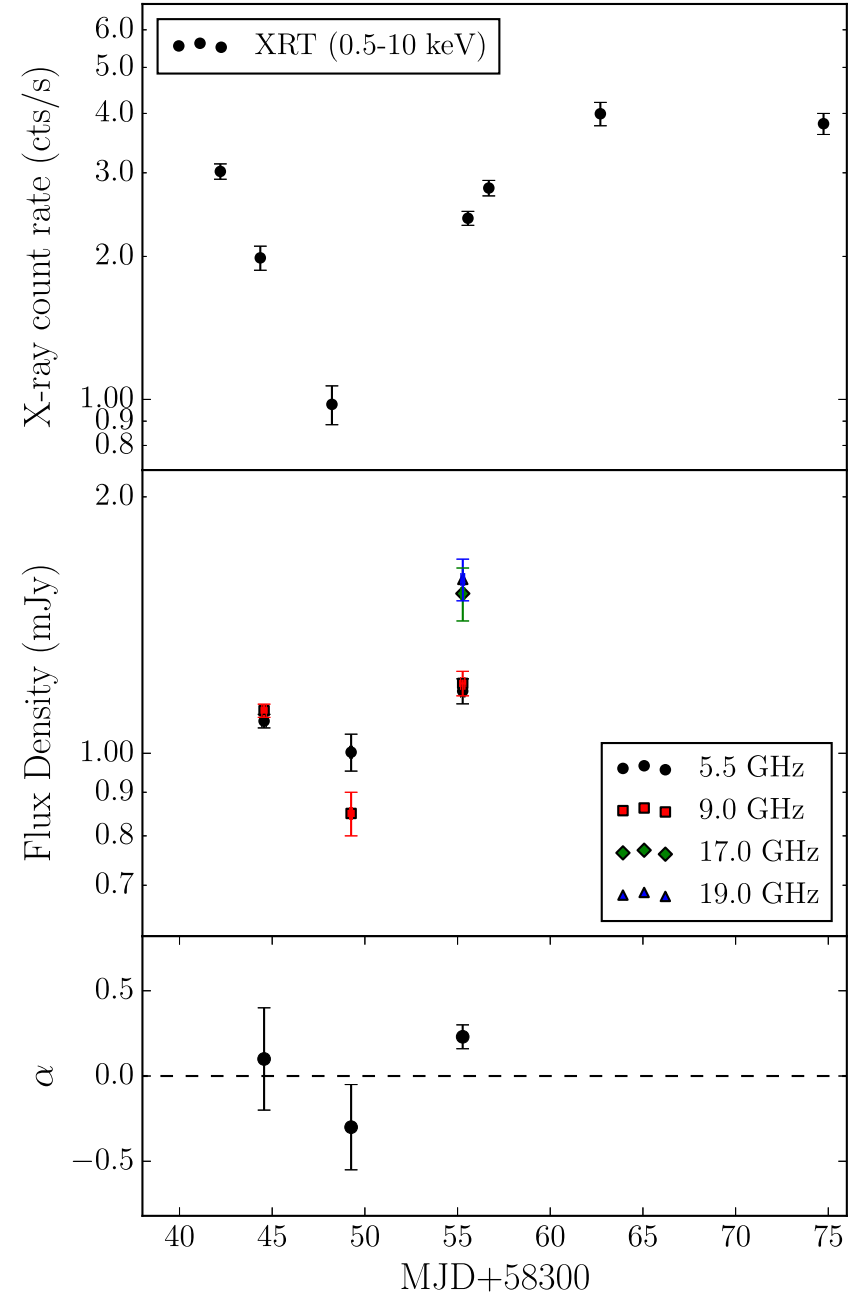

Figure 1. X-ray and radio light curves of IGR J17591-2342 during the early stages of its 2018 outburst. Top panel: $0.5-10 \mathrm{keV}$ Swift-XRT observations. Middle panel: ATCA radio flux densities. Bottom panel: radio spectral indices. Errors are $1-\sigma$.

peak flux of the bursts reached the empirical NS Eddington limit.

\subsection{Absorption along the Line of Sight}

The soft X-ray emission observed from IGR J17591-2342 is highly absorbed. Using interstellar medium (ISM) abundances from Wilms et al. (2000), we find a best fit from Swift-XRT data of $N_{\mathrm{H}}=(4.4 \pm 0.3) \times 10^{22} \mathrm{~cm}^{-2}$. This is consistent with results 


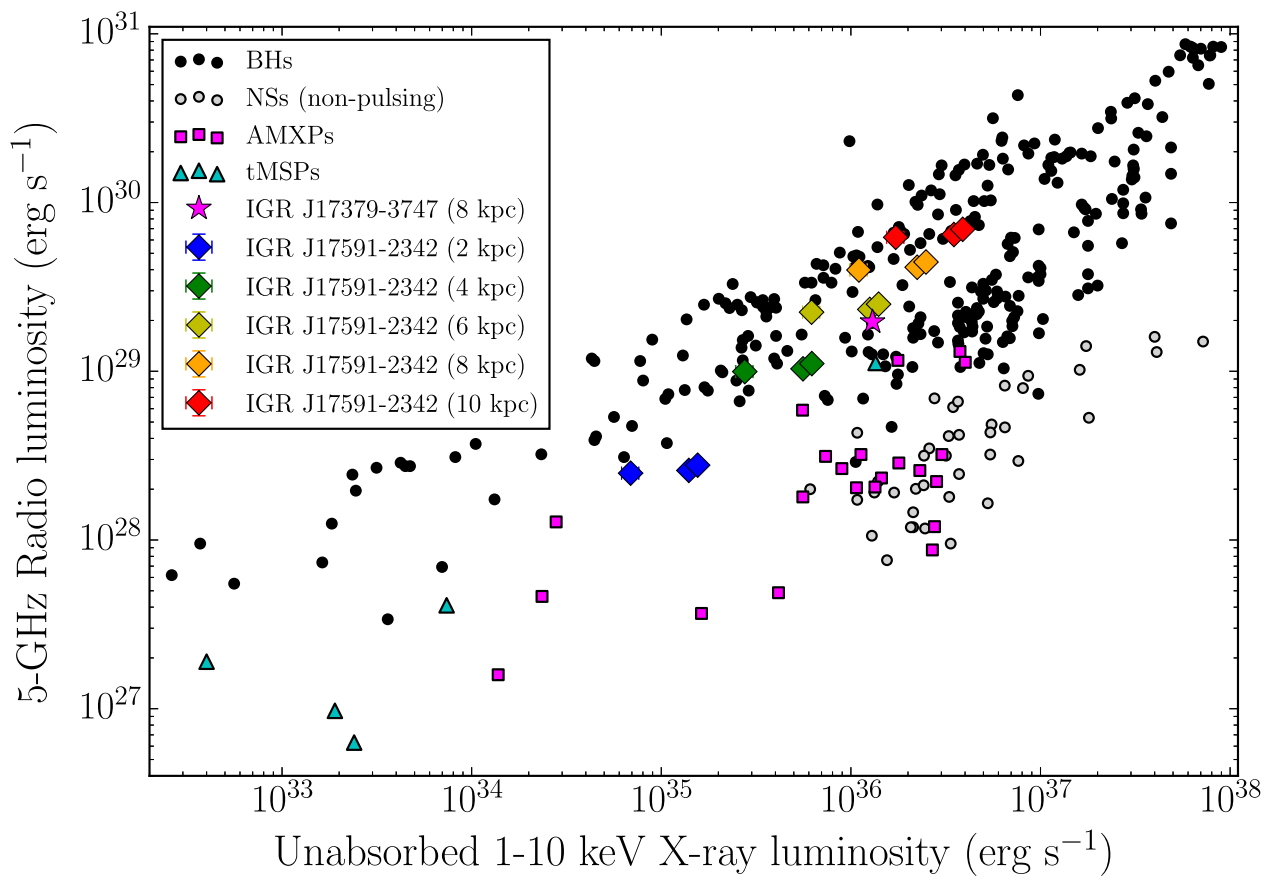

Figure 2. Radio/X-ray measurements of IGR J17591-2342 taken during the early stages of its 2018 outburst. This figure is adapted from Bahramian et al. (2018), and shows the radio/X-ray observations for different types of accreting stellar-mass compact objects (often multiple observations for the same source). IGR J17591 -2342 is shown by the diamonds, where the colors show different assumed distances (see Section 3.2). We also show other AMXPs (magenta squares), highlighting IGR J17379-3747 (magenta star) and tMSPs (cyan triangles). We see that at distances $\gtrsim 3 \mathrm{kpc}$ IGR J17591-2342 appeared more radio luminous than other NS systems at a similar X-ray luminosities. In fact, at such distances, IGR J17591-2342 lies in the region of parameter space usually occupied by black holes.

presented by Sanna et al. (2018), where the $N_{\mathrm{H}}$ was determined to be $(3.6 \pm 1.1) \times 10^{22} \mathrm{~cm}^{-2}$ from INTEGRAL, NuSTAR, and SwiftXRT observations taken at similar times. Our result is marginally higher than that found using the NICER data taken on similar dates (August 15 and August 18), which gave $\left(3.45_{-0.15}^{+0.18}\right) \times 10^{22} \mathrm{~cm}^{-2}$ and $\left(3.59_{-0.08}^{+0.05}\right) \times 10^{22} \mathrm{~cm}^{-2}$, respectively (Sanna et al. 2018), agreeing at the $\sim 2-\sigma$ level. Chandra observations taken on August 23 find $N_{\mathrm{H}}=(4.9 \pm 0.2) \times 10^{22} \mathrm{~cm}^{-2}$ (Nowak et al. 2018), which is consistent within the errors of our measured value.

IGR J17591-2342 lies in the direction of the Galactic center (Ducci et al. 2018), with an unknown distance to the source. However, our modeled $N_{\mathrm{H}}$, which traces the interstellar gas along the line of sight, is $\sim 3-4$ times higher than the expected Galactic contribution of $N_{\mathrm{H}}$ Gal $=1.12-1.44 \times 10^{22} \mathrm{~cm}^{-2}$ (Dickey \& Lockman 1990; Kalberla et al. 2005; Willingale et al. 2013). While a difference between the measured and expected $N_{\mathrm{H}}$ does not indicate the distance to the source and the results may be dependent on the spectral model, it suggests that the source is not nearby. Converting between $N_{\mathrm{H}}$ and optical extinction (Bahramian et al. 2015), we can also compare against higher-resolution three-dimensional optical reddening maps (Green et al. 2018). From these maps we also find that the measured absorption is significantly higher than the expected line-of-sight Galactic reddening, where the total Galactic contribution from reddening maps corresponds to $\sim 2.2 \times 10^{22} \mathrm{~cm}^{-2}$. In fact, along this line of sight, for a distance of $<6 \mathrm{kpc}$, the Galactic contribution is expected to only be $\lesssim 7 \times 10^{21} \mathrm{~cm}^{-2}$.

To further test the relationship with source distance, we compared the $N_{\mathrm{H}}$ Gal (Dickey \& Lockman 1990; Kalberla et al. 2005; Willingale et al. 2013) to the published ${ }^{4} N_{\mathrm{H}}$ for the 28

\footnotetext{
4 To be consistent, when possible, $N_{\mathrm{H}}$ from the literature were determined with Tbabs.
}

BHXRBs and NSXRBs with well-estimated source distances ${ }^{5}$ (Miller-Jones et al. 2014; Gandhi et al. 2018, and references therein). We find that in all cases but two (GRS 1915+105 and V4641 Sgr), the measured $N_{\mathrm{H}}$ was either below or comparable to the expected Galactic line-of-sight absorption for these systems.

GRS $1915+105$ and V4641 Sgr are both atypical and relatively distant BHXRBs $(>6 \mathrm{kpc}$ ), where the measured $\mathrm{X}$-ray absorption exceeds the expected line-of-sight absorption by a factor of $\sim 4-8$ due to anomalously high elemental abundances (Orosz et al. 2001; Lee et al. 2002). For these systems, the overabundances are thought to be related to material in or near the local environment of the source (e.g., Mirabel et al. 1996; Martí et al. 2000; Lee et al. 2002). Other systems with unknown distances also exhibit higher than expected absorption; however, this is generally ascribed to their close proximity to the Galactic center (estimated from the high absorption; e.g., Miller et al. 2006) or are high-inclination "dipping" sources (e.g., Parmar et al. 2002), where disk material or the donor star passes through the line of sight. However, IGR J17591-2342 does not display X-ray dips (Sanna et al. 2018), which are observed from high-inclination systems, implying that inclination is not the cause for the high $N_{\mathrm{H}}$.

There is some debate over the origin of the dominant X-ray absorber for XRBs, whether it be the ISM (even with highly ionized winds from the XRB; (e.g., Miller et al. 2009), or from gas intrinsic to the XRB (e.g., Luo \& Fang 2014). However, from the absorption we favor the scenario in which IGR J17591 -2342 is not nearby $(>6 \mathrm{kpc}$ ), likely close to the Galactic center (where the high absorption may arise from within the Galactic bulge). At such a distance IGR J17591-2342 is very

\footnotetext{
To test for differences between nearby and more distant objects.
} 
radio-loud, with an $L_{\mathrm{R}}$ comparable to those observed from BHXRBs. This result identifies limitations when classifying XRBs from their $L_{\mathrm{R}} / L_{\mathrm{X}}$ ratio. It is important to note that some of the $N_{\mathrm{H}}$ may arise from absorbing material local to the system, similar to what has been proposed for GRS 1915+105 and V4641 Sgr. While this would allow IGR J17591-2342 to be located nearby, we do not expect it to be closer than $\sim 3 \mathrm{kpc}$, thus making this a radio-loud NS. High-resolution X-ray spectroscopy is needed to identify element overabundances from absorption local to the source (Nowak et al. 2018).

\subsection{Why is IGR J17591-2342 Radio Bright?}

While flaring from a transient jet or possibly a propeller mechanism could enhance the observed radio emission (e.g., Tudor et al. 2017), our observations suggest that the radio emission originated from a steady, compact jet.

Our first and third radio epochs showed a flat-to-inverted radio spectrum, inconsistent with emission from a transient jet (which would produce a steep spectrum). While the radio spectrum may have been steep during our second epoch, the flux remained stable over the three epochs, arguing against significant flaring. Additionally, during the first epoch ${ }^{6}$ we did not detect significant intra-observation variability.

Furthermore, using system parameters determined by Sanna et al. (2018) and typical AMXP values, we expect a magnetic propeller regime to occur at an $L_{\mathrm{X}} \lesssim 8 \times 10^{34} \mathrm{erg} \mathrm{s}^{-1}$ (Tsygankov et al. 2017). Therefore, IGR J17591-2342 would need to be at distances $\lesssim 1.5 \mathrm{kpc}$ for the observed radio emission to be propeller driven, which we find unlikely (Section 3.3).

There is thought to be some dependence between jet emission and spin for an accreting NS (Parfrey et al. 2016). IGR J17591-2342 is one of the more rapidly spinning AMXPs (527.4 Hz; Sanna et al. 2018). However, comparing its spin and radio luminosity with other AMXPs, there does not appear to be a clear relationship between these two properties. Therefore, while the NS spin may play some part in enhancing the jet emission in IGR J17591-2342, other characteristics, such as the magnetic field, NS mass, inclination, jet velocity, or launching mechanism, must play an important role in the observed jet emission of other systems (although the magnetic field of IGR J17591-2342 is typical of AMXP systems; see Sanna et al. 2018).

\section{Conclusions}

Using quasi-simultaneous observations, we explore the $L_{\mathrm{R}} / L_{\mathrm{X}}$ of IGR J17591-2342 during the early stages of its 2018 outburst. We find that the source is highly absorbed in the soft X-ray band. Therefore, either IGR J17591-2342 is relatively distant and exceptionally radio-loud, or the source is more nearby with a significant contribution to the absorption from material local to the source, similar to the BHXRBs GRS 1915+105 and V4641 Sgr. If IGR J17591-2342 is at $>3 \mathrm{kpc}$, then our observations place it at radio luminosities more similar to those observed from BHXRBs than NS systems, which could mean that such radio-loud NSs would be a source of confusion when classifying XRBs from their $L_{\mathrm{R}} / L_{\mathrm{X}}$.

This was the only epoch that we could test (Section 2.1).
We thank Jamie Stevens and the ATNF staff for the rapid scheduling of the ATCA observations. T.D.R. thanks Tobias Beuchert, Alice Borghese, Guglielmo Mastroserio, Pikky Atri, Juan Hernández Santisteban and the Jetset group for helpful discussions. T.D.R. acknowledges support from the Netherlands Organisation for Scientific Research (NWO) Veni Fellowship. N.D. and J.v.d.E. are supported by a NWO Vidi grant, awarded to N.D. N.V.G. acknowledges funding from NOVA. J.W.T.H. acknowledges funding from an NWO Vidi fellowship and from the European Research Council under the European Unions Seventh Framework Programme (FP/20072013)/ERC Starting Grant agreement No. 337062 (DRAGNET). J.C.A.M.-J. is the recipient of an Australian Research Council Future Fellowship (FT140101082). The International Centre for Radio Astronomy Research is a joint venture between Curtin University and the University of Western Australia, funded by the state government of Western Australia and the joint venture partners. The Australia Telescope Compact Array is part of the Australia Telescope National Facility which is funded by the Australian Government for operation as a National Facility managed by CSIRO. We acknowledge the use of public data from the Swift data archive. This research has made use of NASA's Astrophysics Data System (ADS).

Facilities: ATCA, Swift-XRT.

Software: CASA, XSPEC.

\section{ORCID iDs}

T. D. Russell (1) https://orcid.org/0000-0002-7930-2276

J. W. T. Hessels (i) https://orcid.org/0000-0003-2317-1446

J. C. A. Miller-Jones (i) https://orcid.org/0000-0003-

3124-2814

\section{References}

Alpar, M. A., Cheng, A. F., Ruderman, M. A., \& Shaham, J. 1982, Natur, 300,728

Archibald, A. M., Stairs, I. H., Ransom, Scott M., et al. 2009, Sci, 324, 1411

Arnaud, K. A. 1996, in ASP Conf. Ser. 101, Astronomical Data Analysis Software and Systems V, ed. G. H. Jacoby \& J. Barnes (San Francisco, CA: ASP), 17

Bahramian, A., Heinke, C. O., Degenaar, N., et al. 2015, MNRAS, 452, 3475

Bahramian, A., Miller-Jones, J., Strader, J., et al. 2018, Radio/X-ray Correlation Database for X-ray Binaries, Version v0.1, Zenodo, doi: 10. 5281/zenodo. 1252036

Blandford, R. D., \& Königl, A. 1979, ApJ, 232, 34

Corbel, S., Coriat, M., Brocksopp, C., et al. 2013, MNRAS, 428, 2500

Dickey, J. M., \& Lockman, F. J. 1990, ARA\&A, 28, 215

Ducci, L., Kuulkers, E., Grinberg, V., et al. 2018, ATel, 11941, 1

Evans, P. A., Beardmore, A. P., Page, K. L., et al. 2009, MNRAS, 397, 1177 Fender, R. P. 2001, MNRAS, 322, 31

Fender, R. P., \& Kuulkers, E. 2001, MNRAS, 324, 923

Gallo, E., Degenaar, N., \& van den Eijnden, J. 2018, MNRAS, 478, L132

Gallo, E., Miller, B. P., \& Fender, R. 2012, MNRAS, 423, 590

Gallo, E., Miller-Jones, J. C. A., Russell, D. M., et al. 2014, MNRAS, 445, 290

Gandhi, P., Rao, A., Johnson, M. A. C., Paice, J. A., \& Maccarone, T. J. 2018, arXiv:1804.11349

Green, G. M., Schlafly, E. F., Finkbeiner, D., et al. 2018, MNRAS, 478, 651 in 't Zand, J. J. M., Heise, J., Muller, J. M., et al. 1998, A\&A, 331, L25

Kalberla, P. M. W., Burton, W. B., Hartmann, D., et al. 2005, A\&A, 440, 775 Krimm, H. A., Barthelmy, S. D., Cummings, J. R., et al. 2018, ATel, 11981, 1 Lee, J. C., Reynolds, C. S., Remillard, R., et al. 2002, ApJ, 567, 1102 Luo, Y., \& Fang, T. 2014, ApJ, 780, 170

Maccarone, T. J. 2008, in ASP Conf. Ser. 401, RS Ophiuchi (2006) and the Recurrent Nova Phenomenon, ed. A. Evans et al. (San Francisco, CA: ASP), 191

Martí, J., Mirabel, I. F., Chaty, S., \& Rodríguez, L. F. 2000, A\&A, 356, 943 Martí-Vidal, I., Vlemmings, W. H. T., Muller, S., \& Casey, S. 2014, A\&A, 563, A136 
McMullin, J. P., Waters, B., Schiebel, D., Young, W., \& Golap, K. 2007, in ASP Conf. Ser. 376, Astronomical Data Analysis Software and Systems XVI, ed. R. A. Shaw, F. Hill, \& D. J. Bell (San Francisco, CA: ASP), 127 Migliari, S., \& Fender, R. P. 2006, MNRAS, 366, 79

Miller, J. M., et al. 2006, ApJ, 646, 394

Miller, J. M., Cackett, E. M., \& Reis, R. C. 2009, ApJL, 707, L77

Miller-Jones, J. C. A., Raymond, J., \& Homan, J. 2014, PASA, 31, e016

Mirabel, I. F., Rodriguez, L. F., Chaty, S., et al. 1996, ApJL, 472, L111

Nowak, M., Paizis, A., Chenevez, J., et al. 2018, ATel, 11988, 1

Orosz, J. A., Kuulkers, E., van der Klis, M., et al. 2001, ApJ, 555, 489

Papitto, A., Ferrigno, C., Bozzo, E., et al. 2013, Natur, 501, 517

Parfrey, K., Spitkovsky, A., \& Beloborodov, A. M. 2016, ApJ, 822, 33

Parmar, A. N., Oosterbroek, T., Boirin, L., \& Lumb, D. 2002, A\&A, 386, 910

Patruno, A., \& Watts, A. L. 2012, arXiv:1206.2727

Radhakrishnan, V., \& Srinivasan, G. 1982, CSci, 51, 1096
Russell, T., Degenaar, N., Wijnands, R., \& van den Eijnden, J. 2018, ATel, 11954, 1

Sanna, A., Ferrigno, C., Ray, P. S., et al. 2018, A\&A, 617, L8

Stappers, B. W., Archibald, A. M., Hessels, J. W. T., et al. 2014, ApJ, 790, 39

Tsygankov, S. S., Mushtukov, A. A., Suleimanov, V. F., et al. 2017, A\&A, 608, A17

Tudor, V., Miller-Jones, J. C. A., Patruno, A., et al. 2017, MNRAS, 470 324

van den Eijnden, J., Degenaar, N., Russell, T. D., et al. 2018a, Natur, 562, 233

van den Eijnden, J., Degenaar, N., Russell, T. D., et al. 2018b, ATel, 11487, 1

Wijnands, R., \& van der Klis, M. 1998, Natur, 394, 344

Willingale, R., Starling, R. L. C., Beardmore, A. P., Tanvir, N. R., \& O'Brien, P. T. 2013, MNRAS, 431, 394

Wilms, J., Allen, A., \& McCray, R. 2000, ApJ, 542, 914 